\title{
Editorial
}

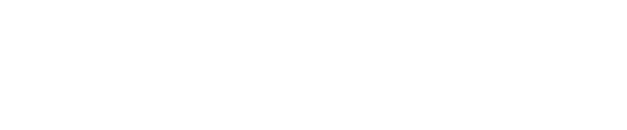

\section{Ayse Kuruüzüm-Uz}

Hacettepe University, Faculty of Pharmacy, Department of Pharmacognosy, Ankara, Turkey
Correspondence:Ayse Kuruüzüm-Uz Hacettepe University, Faculty of Pharmacy, Department of Pharmacognosy, 06100 Ankara, Turkey

$\mathrm{Tel}+903123051089$

$\mathrm{Fax}+903123114777$

E-mail aysekuruuzumuz@gmail.com
Welcome to the first edition of Botanics: Targets and Therapy. This completely open access journal focuses on the discovery and development of active compounds naturally occurring in the plant kingdom which may have therapeutic potential in any disease state.

The aim of the journal is to publish articles on specific topics such as pharmacognosy, identification and isolation of new agents with novel or improved mechanisms of action, clinical utility, and comparison with established or biologic agents, toxicology and tolerability, industrial production; critical evaluation of herbal medicines, patient perspectives, acceptability and satisfaction assessment, and health economics.

Natural products continue to provide a diverse and unique source of bioactive lead compounds for drug discovery, but maintaining their continued importance as source compounds is challenging in the face of the changing pharmaceutical industry. ${ }^{1}$

Despite competition from other drug discovery methods, natural products are still providing their fair share of new clinical candidates and drugs. Natural products are still a significant source of new drugs, especially for anticancer, antimicrobial, and antihypertensive therapies. Therefore, in addition to being a proven and important source of drug leads, drugs derived from natural products also contribute significantly to the profitability of many companies. ${ }^{2,3}$

While the pharmaceutical industry in the developed world will continue to investigate promising leads from natural products in their effort to produce new drugs entities, the production of new medicines in the developing world may have quite different priorities. Here, when a plant is readily available and has the potential to provide inexpensive therapy for the treatment of a disease, a product may well be developed. Close collaboration is expected between clinicians and scientists with a common endeavor - production of safe, quality, and efficacious products. ${ }^{4}$

An estimated 10 to 100 million species or organisms inhabit the earth. Because only $6 \%$ of the 250,000 species of higher plants have been investigated for biological activities and $15 \%$ for their chemical constituents, we have barely scratched the surface of this wonderful resource. ${ }^{4}$

Plants have formed the basis of traditional medicine for thousands of years and continue to provide mankind with new remedies. Modern medicine has its roots in ancient medicine, and it is likely that many important new remedies will continue to be discovered and commercialized in future by following the leads provided by traditional knowledge and experience. Most of the secondary plant compounds submit your manuscript | www.dovepress.com

Dovepress

DOI: $10.2147 /$ BTAT.S1768|
Botanics:Targets and Therapy 20 I I:I I-4

(C) 20II Kuruüzüm-Uz, publisher and licensee Dove Medical Press Ltd.This is an Open Access article which permits unrestricted noncommercial use, provided the original work is properly cited. 
employed in modern medicine were first discovered through ethnobotanical investigation. ${ }^{4}$

Drug discovery is a complex, interdisciplinary pursuit of chemistry, pharmacology, and clinical sciences. ${ }^{2}$ A multidisciplinary subject like pharmacognosy, one of several scientific disciplines bridging biology and chemistry with medicine, has a strategic position for studies of complex scientific questions based on observations in nature. In today's society, we see a renewed interest in "green" chemistry and the sustainable use of natural products. These tendencies are evident also in the search for new pharmaceutical and biotechnological possibilities, where the development of multidisciplinary incentives will probably be necessary. Several different strategies, based on ethnopharmacological, ecological, or toxicological observations together with random screening, and, more recently, phylogenetic frameworks, have been used to prospect this biodiversity in the search for unique structure-activity relationships, and have resulted in revolutionary discoveries in medicine. The ethnopharmacological approach has been the platform for research programs to study plants used in traditional medicine in different cultures. With the increased awareness of the rights of indigenous people to their traditional knowledge, established in several conventions, it has already become increasingly complicated to perform and finance such research. ${ }^{5,6}$

Herbal medicines, in addition to their traditional values, also hold great public and medical interest worldwide as sources of nutraceuticals or novel lead compounds for drug development. A thorough integration of information from genomics, proteomics, and metabolomics is expected to provide solid evidence-based scientific rationales for the development of modern phytomedicines. The search for active phytocompounds will be greatly advanced by the combination of various metabolomics approaches with an array of bioactivity assays in mammalian systems to differentiate between plant species, tissues, or phytopreparations, and to identify novel lead compound candidates for future development. In a complementary development, the use of metabolome-refined herbal extracts with other biochemical components in combination, rather than as isolated single compound(s), may prove to be very useful in providing broader and holistic therapeutic or pharmacological agents for a variety of human health care applications. ${ }^{7}$

All scientific disciplines now recognize the importance of plants as sources of medicines and have initiated research programs either to isolate new lead compounds or to produce standardized extracts. With the interest in natural products from the general public, university researchers, and multinationals across the globe, there is now more than ever a golden opportunity for natural products to make an increasingly worthwhile contribution to health care. ${ }^{4}$

Current research in drug discovery from medicinal plants involves a multifaceted approach combining botanical, phytochemical, biological, pharmacological, and molecular techniques. Medicinal plant drug discovery continues to provide new and important leads against various pharmacological targets including cancer, HIV/AIDS, Alzheimer's, malaria, and pain. Although drug discovery from medicinal plants continues to provide an important source of new drug leads, numerous challenges need to be overcome, including the procurement of plant materials, the selection and implementation of appropriate high-throughput screening bioassays, and the scale-up of active compounds. Isolation and characterization of pharmacologically active compounds from medicinal plants continue today. More recently, drug discovery techniques have been applied to the standardization of herbal medicines, to elucidate analytical marker compounds. $^{8}$

Plant and plant constituents have a key position in the advancement of modern studies and knowledge on biological activity or substances. There are several reasons for this:

- First, plant species, whether traditionally used or not, continue to be important sources of food, medicine, and supplementary health products.

- Second, the bioactive plant compounds are themselves products (or derived products) of metabolism, and hence function in life processes in a similar way to compounds that operate in humans and animals. Researchers hoping to develop drugs from plants need to understand the basics of such functions and mechanisms in relation to the bioactive molecular entities.

- Third, plants also yield products, which are auxiliaries in medicine and pharmacy and sustain or condition pharmacological activity and therapeutic efficacy. In addition, a series of these auxiliary substances are used in biomedical research and in clinical tests. ${ }^{4}$

Plant drugs, also known as phytomedicines, phytopharmaceuticals, or phyto (pharmaco)therapeutic agents, are plant-derived medicines that contain a chemical compound or more usually mixtures of chemical compounds that act individually or in combination on the human body to prevent disorders and to restore or maintain health. ${ }^{4}$

Medicinal plants typically contain mixtures of different chemical compounds that may act individually, additively or in synergy to improve health. Modern medicine usually aims to develop a patentable single compound or a 
"magic bullet" to treat specific conditions. Traditional medicine often aims to restore balance by using chemically complex plants, or by mixing together several different plants in order to maximize a synergistic effect or to improve the likelihood of an interaction with a relevant molecular target. Phytotherapeutic agents are standardized herbal preparations consisting of complex mixtures of one or more plants which contain plant parts or plant material in the crude or processed state as active ingredients.$^{4}$

The term "botanical (or "botanics") is generally used in the broader sense of the word as it would include herbal drugs/extracts and phytochemicals. Phytochemicals are enriched or selected fractions of plant extracts. Currently, the terms are being used for secondary metabolites of plants that may exert health-related effects. Therefore, botanicals are the bioactive ingredients added to or already inherently present in foods and beverages, in order to make them functional. To date, safety concerns, as well as time-consuming efficacy and toxicological studies, have prevented a broader use of herbal ingredients. Prevention-oriented medicine and more self-responsibility in health issues are becoming a political necessity, especially as the population ages. ${ }^{4}$

The renaissance of phytomedicine globally has created a demand for studies on drug discovery from medicinal plants. From a practical perspective, this includes:

- quality (identity, purity, standardization, consistency);

- efficacy (therapeutic indications, clinical studies, pharmacological investigations);

- safety (adverse reactions, drug interactions, contraindications, precautions).

Nowadays, the demands of production, standardization, quality, efficacy, dosage, bioavailability, and safety are placing an increasing emphasis on the use of standardized materials. The chemistry and efficacy of many plants are relatively unknown and there is a chance of toxicity or overdose until the secondary compounds are known and understood. There is a tendency to regulate and license this market, which has led to greater and more effective use of these important medicinal plants. There is also general agreement that chemical standardization is the way forward in order for herbal remedies to be prescribed to patients who seek to be treated with medicinal plants. ${ }^{4}$

It is accepted that concentration or dosages are very important because herbal medicines contain biologically active substances that may produce nontrivial side effects when taken in excessive doses. Very low doses, on the other hand, may have no therapeutic value. In practice, plant material is often highly variable, so that a minimum concentration or a concentration range is often used rather than an exact level. An upper limit is necessary with highly active or potentially harmful ingredients, as most plants have a wide therapeutic window (eg, a toxic dose of a compound is considerably higher than the therapeutic dose). In compounds with a narrow therapeutic window, chemical entities are favored, as opposed to extracts. When these phytodrugs are registered they become a medicine that needs to comply with the basic standards required for all drugs. ${ }^{4}$

Standardization also allows comparison of the clinical effectiveness, pharmacological effects, and side effects of a series of products (eg, against a placebo). Standardized extracts supported by clinical data and quality controls have a role in modern phytotherapy. Standardization is a method of assuring a minimum level of active ingredients in the extract and is becoming increasingly important as a means of ensuring a consistent supply of high-quality phytopharmaceutical products. It can be defined as the establishment of reproducible pharmaceutical quality by comparing a product with established reference substances and by defining minimum amounts of one or several compounds or groups of compounds. In the field of phytomedicines, standardization applies only to extracts. Standards for active ingredients to be used in medicinal products may be found in monographs and/or pharmacopeias. Standardized products provide more security and increase the level of trust people have in herbal drugs. $^{4}$

Testing the biological activity of medicinal or potentially medicinal plant materials demands a special approach. Investigations may be focused on understanding the bioactivity of a compounded plant extract or directed simply at isolating a single bioactive chemical compound. In the latter, results often lead to oversimplification or inaccurate explanations of the bioactivity of extract preparations. On the other hand, thorough studies on single bioactive constituents provide important information for plant drug research. However, the much more complex array of molecular interactions and bioactivity mechanisms that arise from plant extracts represents a much greater and more fascinating scientific challenge. Physicochemical properties and chemical parameters are very important factors affecting biological activity. ${ }^{4}$

Herbal medicines and conventional drug therapies are often taken in combination. Interactions between herbal medicines and conventional drugs include: stimulation or inhibition of drug action by concomitant administration of certain herbs; induction of unexpected side effects by coadministration of herbs; or enhancement of drug side effects by coadministration of certain herbs. The frequency 
of potential interactions between herbal medicines and conventional drug therapies must be evaluated. Physicians and other health care providers should be aware of potential herb-drug interactions and should monitor and inform their patients accordingly.

One of the last breakthroughs in drug discovery was the use of mechanism-based screening for bioassay-guided fractionation. Through continual improvement of screening formats, reagent production, robotics, and data management, mechanism-based screening has since become the mainstay of high-throughput screening. Powerful chromatographic methods in combination with high-throughput screening are now a very efficient way to new leads for drug development. ${ }^{2,4}$

Phytocompound-specific signatures in gene and/or protein expression profiles can also be highly useful in pharmacological standardization, such as their use in "biological fingerprinting" of medicinal plant extracts (ie, bioactivity spectra of phytoextracts or phytocompounds versus their medicinal efficacy in test animal or human systems). Metabolomics approaches using GC-MS, LC-MS, or 2D NMR are effective tools for quality control of medicinal plants or herb medicinal products. In addition, they may provide proof of the toxicology/safety measures of specific phytopreparations after metabolism in test animals/humans. As an alternative to the reductionist approach, plant metabolomics strategies are providing new and important insights for medicinal herb research, linking putative bioactivity with the constituent phytocompounds of herbal medicines. ${ }^{7}$
We are hoping to see in the manuscripts offered to Botanics: Targets and Therapy an overview of some of the challenges and suggested ways in which they can be addressed so that botanics research can remain a viable and productive route to drug discovery

Finally, to succeed, Botanics: Targets and Therapy needs breakthroughs to satisfy the demands of both authors and readers. Dear authors and readers, if you have any comments about the papers in this journal - positive, negative, or providing further information - please do not hesitate to contact me or the Publisher at Dove Medical Press, who is supportive and always available to respond to your comments. I wish to thank the members of our Editorial Board, reviewers, authors, and Dove Medical Press for help in establishing Botanics: Targets and Therapy.

\section{References}

1. Kingston DGI. Modern natural products drug discovery and its relevance to biodiversity conservation. J Nat Prod. 2011;74:496-511.

2. Butler MS. The role of natural product chemistry in drug discovery. J Nat Prod. 2004;67:2141-2153.

3. Cragg GM, Newman DJ, Snader KM. Natural products in drug discovery and development. J Nat Prod. 1997;60:52-60.

4. Gurib-Fakim A. Medicinal plants: Traditions of yesterday and drugs of tomorrow. Mol Aspects Med. 2006;27:1-93.

5. Bohlin L, Göransson U, Backlund A. Modern pharmacognosy: Connecting biology and chemistry. Pure Appl Chem. 2007;79: 763-774.

6. Bohlin L, Göransson U, Alsmark C, Wedén C, Backlund A. Natural products in modern life science. Phytochem Rev. 2010;9:279-301.

7. Shyur LF, Yang NS. Metabolomics for phytomedicine research and drug development. Curr Opin Chem Biol. 2008;12:66-71.

8. Balunas MJ, Kinghorn AD. Drug discovery from medicinal plants. Life Sci. 2005;78:431-441.
Botanics: Targets and Therapy

\section{Publish your work in this journal}

Botanics: Targets and Therapy is an international, peer-reviewed, open access journal focusing on the discovery and development of active compounds based upon or found naturally occurring in the plant kingdom that may have therapeutic potential in any disease state. The manuscript management system is completely online and includes a very

Submit your manuscript here: http://www.dovepress.com/botanics-targets-and-therapy-journal

\section{Dovepress}

quick and fair peer-review system. Visit http://www.dovepress.com/ testimonials.php to read real quotes from published authors. 